\title{
Deficiencias en la labor clínica relacionada con la alimentación infantil
}

\author{
Pilar Torre*
}

Universidad Autónoma Metropolitana Unidad Xochimilco, Departamento de Atención a la Salud, Área Salud y Sociedad, Ciudad de México, México

\section{Resumen}

Se analiza la forma como se llevan a cabo dos procesos clínicos relacionados con la alimentación infantil en las consultas médicas de niños menores de seis meses atendidos en un centro de salud de la Ciudad de México. El primero se refiere a la medición antropométrica de los infantes y el segundo, a la identificación de las modalidades alimentarias. Se requieren acciones correctivas en función de la calidad en la atención a la salud infantil en el sector público.

PALABRAS CLAVE: Alimentación infantil. Antropometría. Modalidad alimentaria.

\section{Clinical deficiencies in infant feeding}

\section{Abstract}

The way in which two clinical procedures related to infant feeding are carried out in medical consultations for children younger than six months in a health center in Mexico City is analyzed. The first one refers to infants' anthropometric measurement, and the second, to feeding modalities' identification. Corrective actions are required based on the quality of children's health care in the public sector.

KEY WORDS: Infant feeding. Anthropometry. Feeding modality.

\section{Introducción}

Al analizar la función de los servicios públicos de salud en la alimentación infantil, mediante un estudio etnográfico de casos de mayor alcance, encontramos dos problemas relacionados con la alimentación infantil, observados en la consulta médica de menores de seis meses en un centro de salud de la Ciudad de México: la baja calidad de la antropometría y la inadecuada identificación de las modalidades de alimentación infantil. ${ }^{1}$
La alimentación infantil puede definirse como el trayecto que recorren las crías humanas desde una fuente única de nutrición, la leche materna —o algún sucedáneo- durante los primeros meses, hasta la alimentación variada propia de cada familia, proceso que requiere aproximadamente dos años, cuando ocurre el destete o su equivalente al quitar el biberón. Diversos elementos de la vida social, cultural, familiar y personal de las madres y sus bebés apuntan hacia diferentes modalidades. Un elemento significativo es el contacto con los servicios de salud, lo que se dice
Correspondencia:

*Pilar Torre

E-mail: ptorre@correo.xoc.uam.mx
Gac Med Mex. 2020;156:463-466

Disponible en PubMed

www.gacetamedicademexico.com CC BY-NC-ND (http://creativecommons.org/licenses/by-nc-nd/4.0). 
acerca de la alimentación infantil en las consultas médicas posnatales en los centros de salud.

La Organización Mundial de la Salud propone la lactancia materna (LM) exclusiva (LME) hasta los seis meses de edad y con alimentación complementaria hasta los 24 meses o más. ${ }^{2,3}$

La disminución en la práctica de la LM en México está documentada hace décadas. ${ }^{4}$ En 2015, la Encuesta Nacional de Niños, Niñas y Mujeres señaló que $94.9 \%$ de los menores de dos años fueron alguna vez amamantados; $51 \%$ de las mujeres lactó al recién nacido en la primera hora posparto; la prevalencia de LME en menores de seis meses fue de $30.8 \%$; la duración media de la LM fue de 10.9 meses; $66.4 \%$ de los menores de dos años utilizó biberón el día anterior y solo $39 \%$ recibió lactancia materna apropiada para la edad. ${ }^{5}$ Las contrapartes de estas cifras representan desafíos para las políticas públicas. En este documento se propone aportar elementos a la discusión social acerca de la crianza, la reproducción y los servicios de salud, y fomentar un diálogo entre la academia y la práctica médica.

\section{Deficiencias en la antropometría}

La primera deficiencia clínica se identificó en la obtención, registro e interpretación de la antropometría infantil, que básicamente se refiere a la edad, el sexo, el peso y la longitud de los bebés, y que está relacionada con el diagnóstico nutricional y el crecimiento infantil. No hay procedimientos estandarizados para realizar las mediciones. ${ }^{6}$ El sexo se asigna al nacer. La edad la preguntan los médicos en repetidas ocasiones. Generalmente, las mediciones fueron realizadas por personal de enfermería antes de la consulta. Los bebés fueron pesados desnudos o vestidos, hasta con cobijas y zapatos; en la báscula apropiada o en la báscula para adultos, cargados sus madres de quien se resta el peso para obtener el peso infantil. Solo un consultorio disponía de infantómetro, el instrumento para medir la longitud; la talla era medida con una cinta métrica flexible que recorría el cuerpo del bebé, sobre la mesa de exploración en paralelo a una cinta métrica colocada sobre el barandal; se podían usar o no topes en la cabeza y los pies. Fue común que las cifras no se anotaran inmediatamente y se cometieran errores numéricos.

Varios médicos mostraron dificultad para comprender el alcance y la relación de los indicadores del estado nutricional del infante: peso para la edad, peso para la longitud y longitud para la edad. Como referencia, algunos médicos usaron las gráficas del
Tabla 1. Modalidades de alimentación infantil en 20 casos

\begin{tabular}{|l|c|}
\hline Modalidad & Casos (n) \\
\hline Lactancia materna exclusiva & 6 \\
\hline Lactancia materna y agua & 2 \\
\hline Lactancia materna y fórmula & 4 \\
\hline Lactancia materna y alimentación variada & 3 \\
\hline Lactancia materna, fórmula y alimentación variada & 2 \\
\hline Fórmula y alimentación variada & 3 \\
\hline Total & 20 \\
\hline
\end{tabular}

programa Control del Niño Sano, otros un cartel de un alimento procesado (Gerber®, Nestlé), otros sus propios parámetros y otros más se declararon incompetentes para la interpretación de los datos.

Durante el periodo de observación, al centro de salud llegaron los estándares antropométricos de la Organización Mundial de la Salud. No se realizó presentación ni capacitación alguna. Se asumía que todos sabían cómo se lleva a cabo la antropometría y que la realizaban correctamente, sin embargo, se observaron serias deficiencias en su calidad y alcance.

\section{Identificación de las modalidades alimentarias}

La otra deficiencia clínica se refiere a la identificación de las modalidades de alimentación infantil. En las consultas, los médicos preguntaban a las madres qué come el bebé o algo equivalente. En dos ocasiones, el médico no preguntó y solo prescribió el modelo alimentario por seguir. Con las respuestas maternas se definió la modalidad en consulta para cada caso.

En entrevistas posconsulta se preguntó a la madre qué comió el bebé el día anterior y se designó la modalidad en entrevista. Se identificaron al menos seis modalidades de alimentación infantil (Tabla 1). Al comparar la modalidad en consulta con la de la entrevista, en la Tabla 2 se observa una coincidencia de $50 \%$. Este nivel de precisión no es suficiente para comprender el hecho alimentario en toda su dimensión ni para enfrentar clínicamente la LM.

Las fórmulas lácteas se usaron desde la estancia hospitalaria para la atención del parto, como se observa en los casos 1 y 2 . La LME se concentró en el grupo de hasta 10 semanas de edad; en adelante, las modalidades se diversificaron hasta una 
Tabla 2. Modalidades de alimentación infantil. Coincidencia entre lo obtenido en consulta y en entrevista a la madre

\begin{tabular}{|c|c|c|c|c|}
\hline \multirow[t]{2}{*}{ Caso } & \multirow{2}{*}{$\begin{array}{c}\text { Edad } \\
\text { (semanas) }\end{array}$} & \multicolumn{2}{|c|}{ Modalidad } & \multirow[t]{2}{*}{ Coincidencia } \\
\hline & & Consulta & Entrevista & \\
\hline 1 & 3 días & LME & $L M+F$ & No \\
\hline 2 & 12 días & LME & $L M+F+A$ & No \\
\hline 3 & 13 días & LME & LME & Sí \\
\hline 4 & 4 & LME & LME & Sí \\
\hline 5 & 4 & $L M+F$ & $L M+F$ & Sí \\
\hline 6 & 4 & LME & $L M+A$ & No \\
\hline 7 & 5 & LME & LME & Sí \\
\hline 8 & 7 & $L M+F^{*}$ & $L M+F$ & Sí \\
\hline 9 & 8 & $L M+F^{*}$ & $L M+A$ & No \\
\hline 10 & 8 & LME & LME & Sí \\
\hline 11 & 8 & LME & LME & Sí \\
\hline 12 & 9 & $L M+F$ & $F+$ té + suero oral $+A$ & No \\
\hline 13 & 10 & LME & LME & Sí \\
\hline 14 & 13 & $L M+F$ & $L M+F+A+$ té & No \\
\hline 15 & 14 & LM + leche entera + avena & $L M+$ leche entera + avena + té $+\mathrm{A}$ & No \\
\hline 16 & 14 & $L M+F$ & $L M+F$ & Sí \\
\hline 17 & 22 & $F+$ té $+A$ & F + té + A + papilla comercial + plátano & No \\
\hline 18 & 22 & F + manzana + plátano & $\mathrm{F}+$ frutas + verduras $+\mathrm{A}$ & No \\
\hline 19 & 23 & $\begin{array}{l}\text { LM + F + caldo de pollo + arroz + chayote } \\
\text { + zanahoria + calabaza + papa + plátano } \\
\text { +manzana + pera + agua }\end{array}$ & $\begin{array}{l}\mathrm{LM}+\mathrm{F}+\text { caldo de pollo + arroz + chayote } \\
+ \text { zanahoria + calabaza + papa } \\
+ \text { plátano + manzana + pera + A }\end{array}$ & Sí \\
\hline 20 & $24^{* *}$ & $L M+$ guayaba + mango + papillas & $L M+$ zanahoria + A + té & No \\
\hline
\end{tabular}

alimentación variada, la mayoría con LM y fórmula. De las 20 madres, 17 ofrecían LM, dos más la habían intentado por dos meses, pero al momento de la entrevista ya no amamantaban; un lactante, prematuro, nunca recibió LM. Es decir, 19 de las 20 madres amamantaron, seis de ellas lo habían conseguido exclusivamente al día de la consulta, dos más con agua y nueve combinaban la LM con fórmula láctea y diversos alimentos. La precisión médica para identificar las modalidades de alimentación infantil resultó baja.

\section{Discusión}

En cada consulta se comentó la alimentación y nutrición infantil. Los datos antropométricos están siempre presentes y resultan fundamentales. La calidad de la antropometría fue deficiente y la capacidad del personal médico para diagnosticar el estado de nutrición fue limitada, lo cual merece la atención de las autoridades sanitarias.

La pregunta médica ¿qué come el bebé? es rutinaria y superficial. En dos casos, no se formuló. La precisión descriptiva de las modalidades de alimentación infantil indica que los médicos no realizan las preguntas pertinentes. El caso 2 ilustra que la pregunta tiene una respuesta, pero no se describe la modalidad de la alimentación: el médico preguntó a la madre si había estado dando pecho, a lo que la ella respondió afirmativamente; la modalidad en realidad era LM, fórmula y agua a los 12 días de edad 
infantil, sin embargo, el médico asumió que se trataba de LME.

Pocos médicos aclaran la relación entre la demanda y la producción de leche materna o cómo lograr un buena vinculación entre un bebé que succiona y una madre que produce y ofrece leche a través del amamantamiento. El caso 8, de siete semanas de edad, es extremo: por un lado, el médico instruyó a la madre sobre alimentar al bebé exclusivamente con leche materna hasta los cuatro meses de edad, pero, por otro lado, le indicó que puede alimentar con fórmula si no producía suficiente leche materna.

Hay argumentos para señalar la existencia de serios problemas en el alcance de las prácticas y las prescripciones médicas acerca de la alimentación infantil, los cuales requieren una revisión a la luz del conocimiento actual y los consensos internacionales. Los problemas identificados reflejan nociones arcaicas de la alimentación infantil, medicalizadas e insuficientes para ofrecer consejería a la población que acude a las consultas posnatales, lo cual puede mejorar considerablemente con información, capacitación y voluntad política.

La situación de México es resultado de prácticas médicas, sociales y familiares asentadas en la "cultura del biberón", a la que se refiere la Declaración de Innocenti y la cual es necesario reconocer y describir para oponer argumentos hacia la "cultura de la lactancia". ${ }^{7}$ Respecto a las decisiones cotidianas que conllevan las prácticas de alimentación infantil, las madres interactúan con los servicios de salud, donde se expresan las recomendaciones de la normatividad dietética medicalizada. Podemos argumentar que prevalece gran ignorancia en cuanto a las condiciones y requerimientos de la LM, particularmente en su modalidad exclusiva, y la orientación para llevarla a cabo. La Organización Mundial de la Salud cuenta con modelos de capacitación de gran impacto. ${ }^{8}$ Tiempo atrás, el Hospital General de México operó un centro de capacitación en LM, que pudiera ser el antecedente de acciones a nivel nacional.

\section{Nota necesaria}

En este texto se utiliza el término médico sin distinción del género, más en el sentido de describir la función profesional en la consulta médica, que de nombrar al sujeto, hombre o mujer que la ejerce. Algo similar ocurre con el término bebé.

\section{Agradecimientos}

A Monserrat Salas, por el diálogo teórico-metodológico y su amistad. A Adriana Chávez, por su eficacia. A la Universidad Autónoma Metropolitana Unidad Xochimilco, por su generosidad y ambiente.

\section{Conflicto de intereses}

La autora declara no tener conflicto de intereses alguno.

\section{Financiamiento}

La investigación se realizó con el presupuesto anual para proyectos de investigación que otorga la Universidad Autónoma Metropolitana Unidad Xochimilco (aproximadamente USD 800/año).

\section{Bibliografía}

1. Torre P. Epistemología de la alimentación infantil en los servicios de primer nivel de atención a la salud. Estudio de casos en México. [Tesis doctoral]. País Vasco: Universidad del País Vasco; 2015.

2. Organización Mundial de la Salud. Estrategia Mundial para la Alimentación del Lactante y del Niño Pequeño. Suiza: Organización Mundial de la Salud; 2001.

3. World Health Organization. Optimal duration of exclusive breastfeeding. A systematic review. Suiza: World Health Organization; 2002.

4. Ysunza A. El abandono de la lactancia materna en México I: tendencias recientes. Rev Invest Clin. 1986;38S:97-102.

5. Fondo de las Naciones Unidas para la Infancia/Instituto Nacional de Salud Pública. Encuesta Nacional de Niñas, Niños y Mujeres 2015. Informe final. México: UNICEF/INSP; 2016.

6. Peláez M, Torre P, Ysunza A. Elementos prácticos para el diagnóstico de la desnutrición. México: Instituto Nacional de Ciencias Médicas y Nutrición Salvador Zubirán; 1993.

7. World Health Organization/United Nations International Children's Emergency Fund. The Innocenti Declaration. Breastfeeding in the 1990's. A Global Initiative. Italia: WHO/UNICEF; 1990.

8. Organización Mundial de la Salud/Fondo de las Naciones Unidas para la Infancia. Consejería en lactancia materna. Curso de capacitación. Manual del participante. Suiza: OMS/UNICEF; 1993. 